\title{
Investigation of Relationship Between Hypoxic Process and Cancer Stem Cells in Meningioma
}

\author{
Ismail SAYGIN, Emel CAKIR, Mustafa Emre ERCIN \\ Karadeniz Technical University, Faculty of Medicine, Department of Pathology, Trabzon, Turkey \\ Corresponding author: Ismail SAYGIN ismailsaygin@ktu.edu.tr
}

\section{ABSTRACT}

AIM: To investigate the relationship between cancer stem cells (CD133 and CD44) and HIF1a expression in meningioma.

MATERIAL and METHODS: In an immunohistochemistry experiment, three expert pathologists examined 100 meningioma slides stained for HIF1a, CD133, and CD44 antibodies. Statistical analyses were performed using the SPSS 23 Statistics packet program. $P$ values $<0.05$ were statistically significant.

RESULTS: HIF1a staining was correlated with high grade (Grade 2) meningioma. Cytoplasmic staining was negatively correlated with meningioma grade. High grade meningioma was positive for nuclear HIF1a and showed increased cytoplasmic expression of CD44 and CD133.

CONCLUSION: In accordance with previous studies, the level of hypoxia and HIF1a were correlated with meningioma grade. Furthermore, expression of HIF1a was correlated with expression of CD133 and CD44, which are cancer stem cell surface markers, as well as with meningioma grade. In light of these data, new treatment modalities related to CD44 and CD133 stem cell markers and to HIF1 a may be developed.

KEYWORDS: Hypoxia, HIF1a, CD133, CD44, Cancer stem cell, Meningioma

\section{INTRODUCTION}

M eningiomas, which derive from arachnoid cap cells, are the most common primary tumors of the central nervous system (11). Grade 1 meningiomas have a better prognosis than Grade 2 and 3 meningiomas, which can demonstrate aggressive behavior and have an increased rate of recurrence (10).

Cancer stem cells (CSCs) are rare immortal cells found in tumors that have the ability to regenerate spontaneously. These cells have attracted attention as targets for cancer treatment. The main characteristics of CSCs are as follows: having the ability to self-renew, exhibiting rapid division and proliferation, causing tumor heterogeneity, forming a small population in tumor tissue, playing a role in tumor metastasis, and showing resistance to chemotherapy and radiotherapy. Given these characteristics, there are strong correlations between CSCs and poor prognosis, metastasis, and recurrence (22). In order to target CSCs more effectively, it is important to focus on hypoxic areas that act like stem cell production sites within the tumor.

Hypoxia, which is the most common cause of cell injury, is also one of the most important micro-environmental factors involved in carcinogenesis (16). Increased metabolic requirements and decreased physiological oxygen levels due to rapid proliferation are the characteristics of solid tumors. Hypoxia in the micro-environments of tumors has been shown to cause genetic instability in cancer cells, and to play an important role in the development of the invasive abilities and aggressive characteristics of tumors as well as in their resistance to radio/chemotherapy and the development of metastasis $(24,25)$. Therefore, determining the presence and level of hypoxia in tumor micro-environments has become increasingly important. 
Saygin I. et al: Hypoxia, Cancer Stem Cells and Meningioma

Hypoxia inducible factors (HIFs) act as hypoxia sensors around the tumor micro-environment. HIF proteins regulate and perform the transcription of hypoxia-inducible genes to increase the survival potential of cells during oxygen depletion; thus, these proteins contribute to tumor progression and aggression $(12,21)$. HIFs are currently classified into three groups: HIF1, HIF2, and HIF3. HIF1 plays an important role in maintaining the oxygen balance at both the cellular and systemic levels. It is also the key regulator of the transcriptional activation responses of many genes to hypoxia in various processes such as cell survival and proliferation, angiogenesis, erythropoiesis, glucose intake, and iron metabolism. HIF1, which is found in all types of cells, is a heterodimer consisting of alpha (HIF1 $\alpha$ ) and beta (HIF1 $\beta$ ) subunits (8). Oxygen levels affect protein stabilization, intracellular localization, and transcription of HIF1a; conversely, the HIF1 $\beta$ subunit, which is structurally expressed, is not affected by oxygen levels (2). A HIF-1a protein with a half-life of 5 minutes becomes almost absent in the environment following rapid degradation under normoxic conditions; under hypoxic conditions however, a HIF1a protein such as this does not undergo degradation and is therefore present at high levels. Increased HIF1a proteins subsequently translocate to the nucleus to be heterodimerized with the HIF1 $\beta$ subunit. Thus, HIF1 a acquires transcriptional activity $(3,14)$.

Since hypoxia increases the genetic instability that leads to tumor growth, the HIF system has attracted substantial attention as a target in cancer therapeutics. Clinically, HIF1a can be used as a marker for over expression, high aggressiveness, and treatment challenges.

CD44 and CD133 are cell surface markers and cell adhesion molecules expressed in almost all human cells. Recently, they have been considered as CSCs markers and are associated with a cancer initiating subpopulation of cancer cells. CD44 is crucial for cell proliferation, migration, angiogenesis, inflammation, cell signaling, and cell adhesion (17). In addition, CD44 plays an important role in the communication of CSCs with the micro-environment and in the regulation of stem cell characteristics. CD133 is important for tumor formation, metastasis, anti-apoptosis, and chemotherapy and radiotherapy resistance (27).

The aim of the present study was to reveal the relationship between HIF1a, which is thought to increase during the hypoxic process, and CSCs (CD133 and CD44) in Grade 1 and 2 meningiomas.

\section{MATERIAL and METHODS}

This study included a total of 100 cases including low grade (Grade 1) and high grade (Grade 2/atypical) meningiomas diagnosed between 2008 and 2018. An immunohistochemical study was performed with HIF1a polyclonal antibody (1:200; Elabscience, USA), CD44 monoclonal antibody (1:500; Elabscience, USA), and CD133 polyclonal antibody (1:200; Elabscience, USA) using Ventana's BenchMark Ultra automated system. Slides were evaluated using the Olympus BX51 light microscope. A DAP detection kit (Roche, USA) was used. The research protocol was approved by institutional review board (KTU-24237859-641/19.11.2017).

\section{Immunohistochemical Evaluation}

The results of the immunohistochemical study were evaluated by three pathologists. Cytoplasmic and nuclear stainings were interpreted separately. The intensity and percentage of staining on each slide was randomly evaluated at five different highpower fields (HPFs) (x400 fold magnification) for all antibodies. Staining intensity was scored semi-quantitatively as follows: 0 (no staining), 1+ (weak staining), 2++ (moderate staining), and $3+++$ (strong staining). The percentage of staining was calculated as the ratio of the number of cells stained in one HPF to the total number of cells within the same area.

An $\mathrm{H}$ score formula $(3 \times 3+++$ cell percentage $+2 \times 2++$ cells percentage $+1 \times 1+$ cell percentage $+0 \times$ non-stained cell percentage) was used to evaluate the staining percentages and intensities. Five different $\mathrm{H}$-scores $(0$ to 300$)$ were obtained for each slide using the $\mathrm{H}$ score formula $(5,13)$.

\section{Statistical Analysis}

The SPSS 23.0 statistical package program was used to analyze data. Number and percentage were used as descriptive statistics for categorical data, whereas mean, standard deviation, minimum, and maximum were used for interval data. The interval data were analyzed using the One-Sample Kolmogorov-Smirnov test in terms of normal distribution. For the comparison of two independent groups from the interval data, Student's t-test was used when there was normal distribution and the Mann Whitney $U$ test was used when normal distribution could not be obtained. Comparative analysis was performed by calculating the mean of five different $\mathrm{H}$ scores. The capacity of $\mathrm{H}$ scores for predicting Grade 1 and 2 meningiomas was analyzed using Receiver Operating Characteristics (ROC) curve analysis. The areas under the curve were represented with a 95\% confidence interval $(\mathrm{Cl})$. When a significant cut-off value was observed, the sensitivity and specificity values were presented. A $p$ value $<0.05$ was considered to show a statistically significant result.

\section{RESULTS}

In this study, 53 meningioma cases were Grade 1 whereas 47 were Grade 2. Four of the Grade 1 cases were angiomatous, 16 were fibrous, 17 were transitional, and 16 were meningothelial type. Cytoplasmic and nuclear staining of HIF1a, CD133, and CD44 antibodies are shown in Figure 1A-O; mean $\mathrm{H}$ scores calculated for these stainings are shown in Figure 2. The $\mathrm{H}$ score for cytoplasmic staining of HIF1a was higher in Grade 1 patients (93.5) than in Grade 2 patients (71.4). In contrast, the $\mathrm{H}$ score for nuclear staining of HIF1a was significantly lower in Grade 1 cases (170.9) than in Grade 2 cases (246.1). The $\mathrm{H}$ scores for cytoplasmic and nuclear staining of CD133 and CD44 antibodies increased as the meningioma grade increased. However, the mean $\mathrm{H}$ score for nuclear staining of CD44 was $0.01 \%$ in Grade 1 meningioma and $0.2 \%$ in Grade 2.

The comparative distribution of cases according to $\mathrm{H}$ score threshold values (HIF1a nuclear, CD133 cytoplasmic-nuclear, 


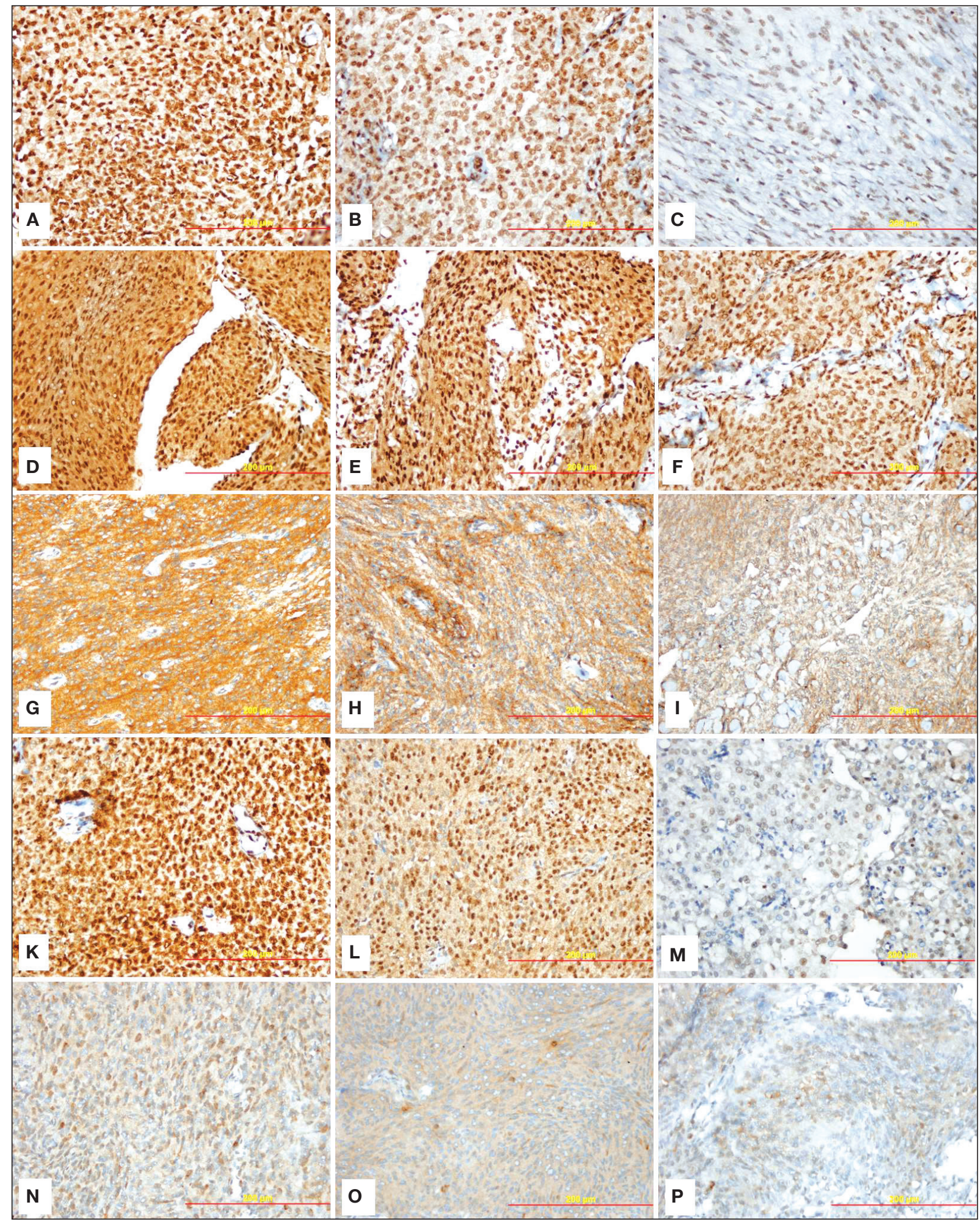

Figure 1: Cytoplasmic and nuclear staining of HIF1a, CD133, and CD44 antibodies. HIF1a (A-F); A: Strong nuclear staining, B: Moderate nuclear staining, C: Weak nuclear staining, D: Strong cytoplasmic staining, E: Moderate cytoplasmic staining, F: Weak cytoplasmic staining. CD133 (G-M); G: Strong cytoplasmic staining, H: Moderate cytoplasmic staining, I: Weak cytoplasmic staining, K: Strong nuclear staining, L: Moderate nuclear staining, M: Weak nuclear staining. CD44 (N-P); N: Strong cytoplasmic staining, O: Moderate cytoplasmic staining, P: Weak cytoplasmic staining. 
and CD44 cytoplasmic) and grades is shown in Figure 3. The $\mathrm{H}$ score threshold value for HIF1a was 217. For HIF1a nuclear staining, 12 (23.5\%) of the cases with an $\mathrm{H}$ score threshold $\geq 217$ were Grade 1 and 39 (76.5\%) were Grade 2. ROC analysis curves used to determine tumor grades for HIF1a, CD133, and CD44 antibodies are shown in Figure 4. The cytoplasmic staining score of HIF1a was not significant in distinguishing the grades and remained below the reference line $(p=0.064)$; the area under the curve was 0.392 (95\% Cl: 0.281-503). The nuclear HIF1 a score was statistically significant in distinguishing the grades $(p<0.001$; sensitivity $=83 \%$; specificity $=77.4 \%)$; the area under the curve was 0.850 (95\% Cl: $0.775-925)$. The

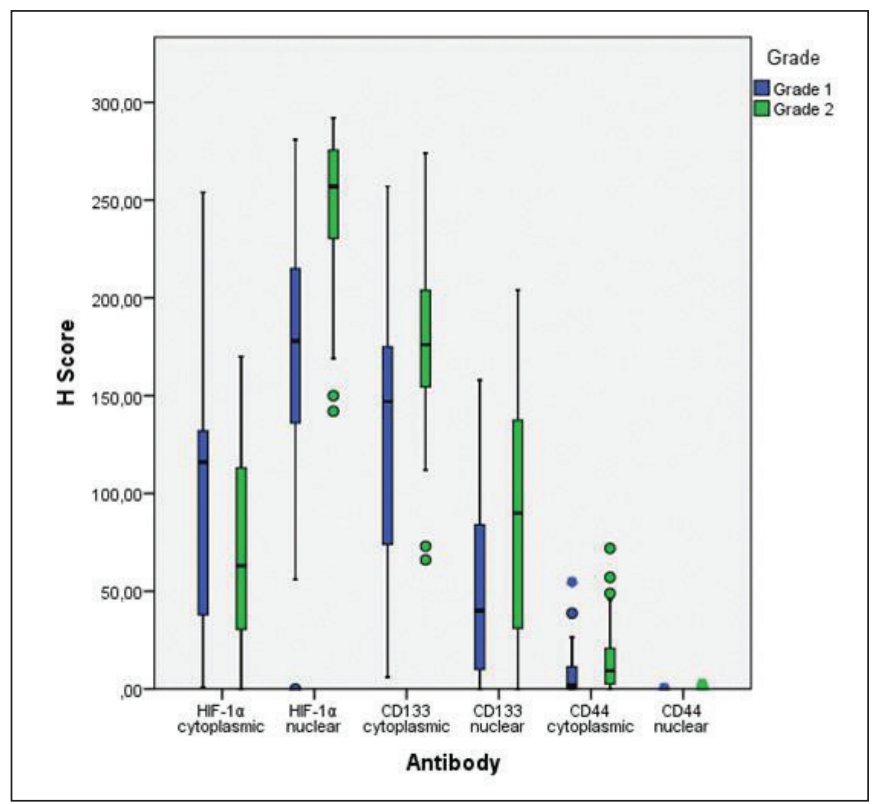

Figure 2: Mean $\mathrm{H}$ scores for cytoplasmic and nuclear staining of HIF1a, CD133, CD44 antibodies and comparative with grade. cytoplasmic CD133 score was also statistically significant in distinguishing the grades $(p<0.001$; sensitivity $=72.3 \%$; specificity $=66 \%)$; the area under the curve was $0.723(95 \%$ Cl: 0.624-821). Additionally, the nuclear CD133 score was statistically significant in distinguishing the grades $(p=0.002$; sensitivity $=66 \%$; specificity $=54.7$ ); the area under the curve was 0.682 (95\% Cl: 0.577-787). The cytoplasmic CD44 score was also statistically significant in distinguishing the grades $(p=0.003$; sensitivity $=72.3$; specificity $=56.6)$; the area under the curve was 0.675 (95\% Cl: 0.570-780). Finally, the nuclear CD44 score was not statistically significant in distinguishing the grades $(p=0.082)$; the area under the curve was 0.601 (95\% Cl: 0.489-714).

The distribution of cases with high HIF1a nuclear $\mathrm{H}$ scores according to the $\mathrm{H}$ score threshold values of CD133 cytoplasmic-nuclear and CD44 cytoplasmic staining are shown in Figure 5. The cytoplasmic staining scores of CD44 $(n=28)$ and CD133 $(n=28)$ were above their thresholds and were of equal number in Grade 2 cases. In addition to the high cytoplasmic staining score of CD133, the nuclear staining score was also high in Grade 2 cases.

\section{DISCUSSION}

In the present study, cytoplasmic staining of HIF1a was negatively correlated with meningioma grade, whereas nuclear staining of HIF1a was positively correlated with meningioma grade. Additionally, hypoxia was found to increase as the grade of meningioma increased. Specifically, as meningioma grade increased the intensity and percentage of HIF1a nuclear staining, considered an immunohistochemical marker of hypoxia, also increased. Here, HIF1a nuclear staining was a significant variable for distinguishing meningioma grade; nuclear staining occurred at higher levels in Grade 2 meningioma, which is compatible with the findings of Reszec et al. (19). The results of the present study showed

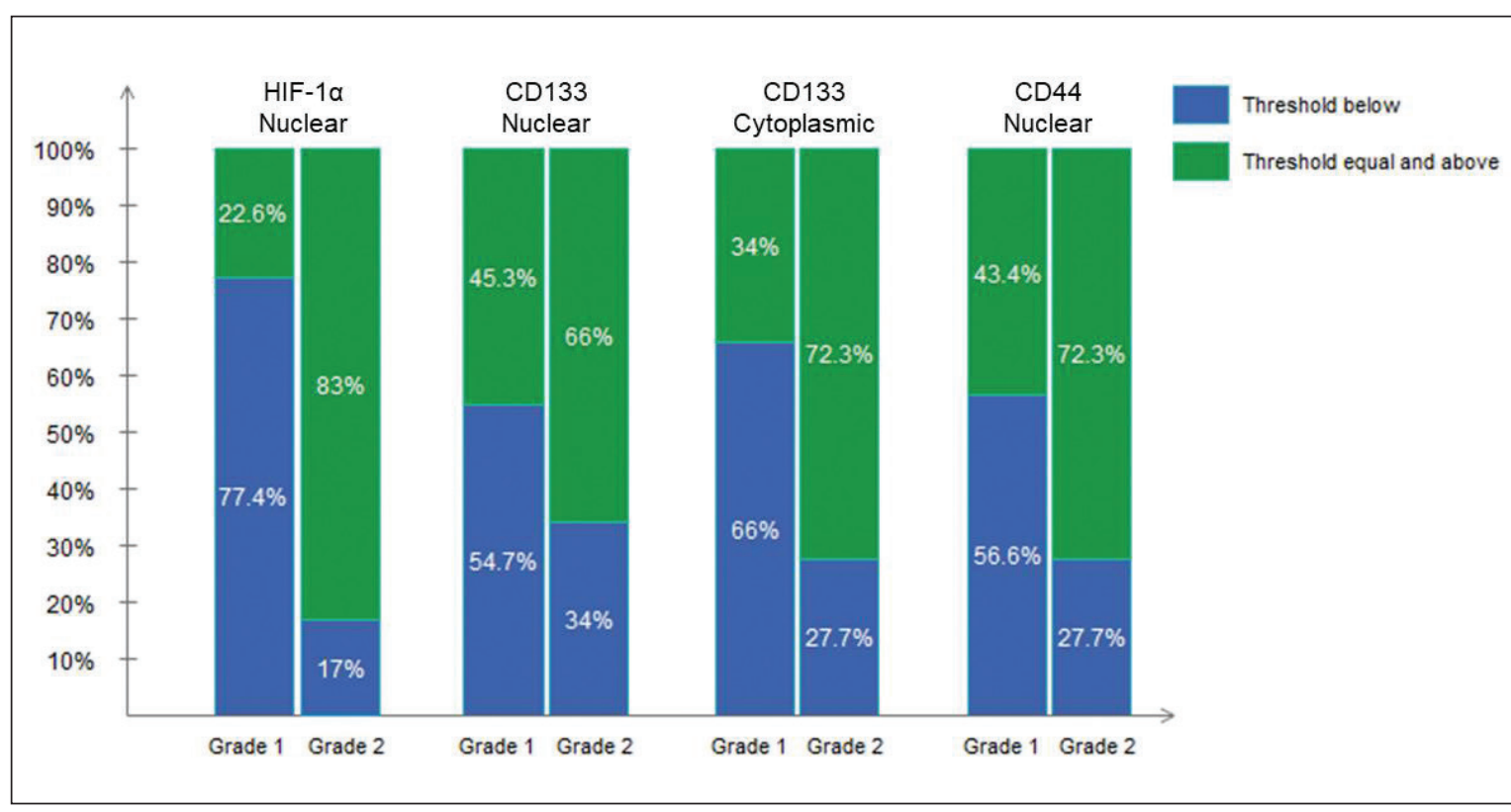

Figure 3: Comperative distribution of cases according to $\mathrm{H}$ score threshold values (HIF1a nuclear, CD133 cytoplasmicnuclear, CD44 cytoplasmic) and grades. 
that, in contrast to HIF1a nuclear staining, HIF1 a cytoplasmic staining was an ineffective variable for differentiating Grade 1 and 2 meningiomas. In another study by Reszec et al., HIF1a antibody was associated with recurrence and higher tumor grades in meningioma and was therefore suggested to be useful for estimating survival in meningioma cases (18). Similarly, Victorio et al. showed that the level of HIF1a expression in intracranial meningioma was associated with recurrence and higher tumor grades (26).

In our study, both cytoplasmic and nuclear stainings of CD133 were observed; moreover, $\mathrm{H}$ scores for both types of staining were correlated with meningioma grade. Here, $\mathrm{H}$ scores for cytoplasmic staining of CD133 antibody increased

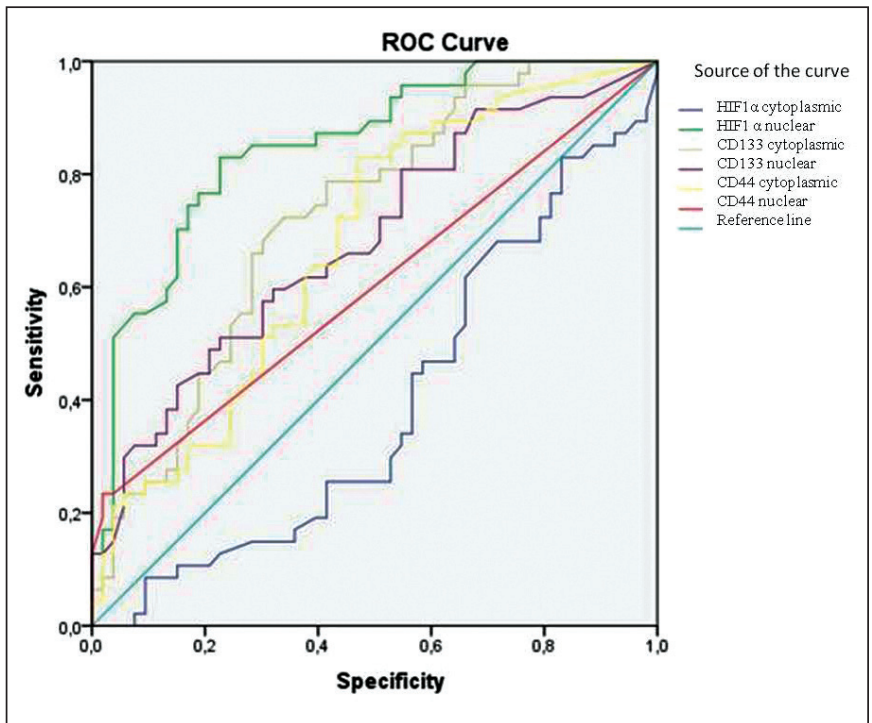

Figure 4: ROC analysis curves used to determine tumor grades for HIF1a, CD133, and CD44 antibodies. as meningioma grade increased. Similarly, in the studies of Xiao and Al-Saka, CD133 was correlated with meningioma grade; these authors emphasized that CD133 was important for demonstrating aggressive behavior and progression in meningioma cases $(4,23)$. In our analysis, nuclear staining of CD133 antibody was also correlated with meningioma grade; statistically, CD133 nuclear staining was a significant variable for distinguishing between Grade 1 and 2 meningiomas. To the best of our knowledge, previous studies on the nuclear staining of CD133 in meningioma have not been published. However, Cantile et al. reported that nuclear staining of CD133 could be observed in various tumor types. They indicated that nuclear localization of CD133 might be an indicator of poor prognosis in triple-negative breast cancer. They also suggested that when CD133 was localized in the nucleus, it might interfere with the molecular pathways that directly affect the proliferation and differentiation of tumor cells and thereby act as a transcription regulator (1). In a study by Huang et al., high levels of CD133 expression were found in both the nucleus and cytoplasm of non-small-cell lung carcinoma, and nuclear localization was associated with poor prognosis (7).

Although the $\mathrm{H}$ score for cytoplasmic staining of CD44 antibody was low in our study, it was correlated with the higher grade meningioma, similar to HIF1a and CD133. Specifically, in Grade 2 meningioma, the $\mathrm{H}$ score for cytoplasmic staining of CD44 was twice that of Grade 1 cases; thus, CD44 cytoplasmic staining was also a significant variable for distinguishing Grade 1 and 2 meningiomas. Similar to our study, Lewy et al. showed strong cytoplasmic expression in atypical meningiomas with the CD44 antibody, and they reported statistically significant results for distinguishing between Grade 1 and Grade 2 cases (9). In other studies, Sainio et al. found evidence that increased expression of CD44 was associated with anaplasia and metastasis in meningioma, whereas Mostafa et al. found that, in 40 cases, higher CD44 expression and the Ki67 proliferation

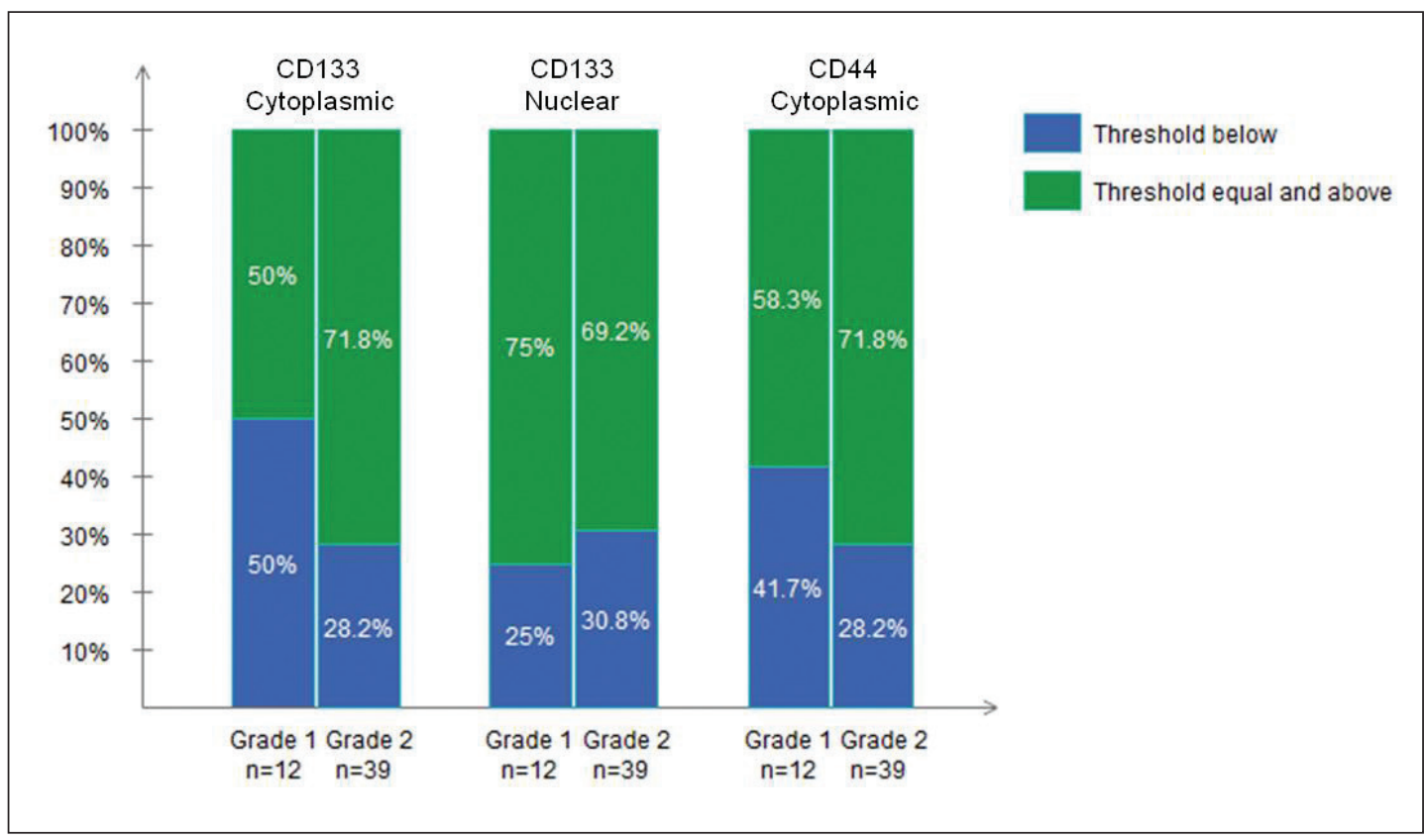

Figure 5: Distribution of cases with high HIF1a nuclear $\mathrm{H}$ scores according to the $\mathrm{H}$ score threshold values of CD133 cytoplasmicnuclear and CD44 cytoplasmic staining. 
index were correlated in high-grade meningioma rather than low-grade meningioma $(15,20)$.

Nuclear staining of CD44 is generally not expected, and in our study nuclear staining of CD44 was indeed weak. Accordingly, the $\mathrm{H}$ score for CD44 antibody nuclear staining in all meningiomas was $<1 \%$. As stated by McCarty, cases with $\mathrm{H}$ scores $<1 \%$ should be considered negative $(5,13)$. Statistically, the $\mathrm{H}$ score for CD44 nuclear staining was therefore an ineffective variable for distinguishing between Grade 1 and 2 meningiomas.

In the present study, the various histological types of Grade 1 meningioma had no significant differences among their $\mathrm{H}$ score mean values for HIF1a, CD44, and CD133. In angiomatous meningiomas only, the $\mathrm{H}$ score for cytoplasmic staining of CD133 was below the threshold value. In contrast to our study, Gassoum et al. found that most fibrous meningiomas showed CD44 expression; our results indicated no significant differences among the $\mathrm{H}$ scores for CD44 in morphological subtypes of meningioma (6).

Although CSC markers and HIF1a have previously been studied in many tumors and meningiomas, the relationship between high $\mathrm{H}$ scores for HIF1a ( $\geq \mathrm{H}$ score threshold value) and CSCs had yet to be studied. Here, cytoplasmic staining of CD133 and CD44 correlated with increased nuclear staining of HIF1a in higher grade hypoxic tumors. This result reveals that there is a high rate of CSCs in the hypoxic environment indicated by HIF1a nuclear staining.

In the present study, cases with high $\mathrm{H}$ scores for HIF1a nuclear staining were evaluated according to meningioma grade. In Grade 2 meningiomas, most of the cases with high $\mathrm{H}$ scores for nuclear staining of HIF1a also had high $\mathrm{H}$ scores for cytoplasmic staining of CD133 and CD44. Furthermore, cytoplasmic staining scores for CD44 and CD133 were similar in Grade 2 cases. Thus, high H scores for HIF1 nuclear staining and CD44/CD133 cytoplasmic staining are apparently typical in Grade 2 meningioma cases.

\section{CONCLUSION}

HIF1a expression correlated with both meningioma grade and the expression of CD133 and CD44 CSC surface markers. We suggest that advanced clinicopathological studies are conducted to analyze the relationships among the expression of HIF1a, CD133, and CD44 and the therapeutic response, survival, and prognosis in meningioma cases.

\section{ACKNOWLEDGEMENTS}

The authors thank Karadeniz Technical University, Scientific Research Project Funding (KTU BAP, Project no: THD-20187199) for their financial support.

\section{REFERENCES}

1. Cantile M, Collina F, D'Aiuto M, Rinaldo M, Pirozzi G, Borsellino C, Franco R, Botti G, Di Bonito M: Nuclear localization of cancer stem cell marker CD133 in triple-negative breast cancer: A case report. Tumori 99(5):e245-250, 2013

2. Chee NT, Lohse I, Brothers SP: mRNA-to-protein translation in hypoxia. Mol Cancer 18(1):49, 2019

3. Depping R, Steinhoff A, Schindler SG, Friedrich B, Fagerlund R, Metzen E, Hartmann E, Köhler M: Nuclear translocation of hypoxia-inducible factors (HIFs): Involvement of the classical importin alpha/beta pathway. Biochim Biophys Acta 1783(3):394-404, 2008

4. El-Saka AM, Zamzam YA: Stem cell markers CD133, MMP-9, and Ki-67 expressions in different grades of meningiomas and their prognostic significance. Egypt J Pathol 33(2):151-156, 2013

5. Fedchenko N, Reifenrath J: Different approaches for interpretation and reporting of immunohistochemistry analysis results in the bone tissue - a review. Diagn Pathol 9:221, 2014

6. Gassoum A, Arbab MA, Aldeaf SAH, Elhassan LA, Bashier BM: CD44 expression in Sudanese meningioma patients. Int J Recent Sci Res 7(6):11900-11904, 2016

7. Huang M, Zhu H, Feng J, Ni S, Huang J: High CD133 expression in the nucleus and cytoplasm predicts poor prognosis in nonsmall cell lung cancer. Dis Markers 2015:986095, 2015

8. Lee HJ, Jung YH, Choi GE, Kim JS, Chae CW, Han HJ: Role of HIF1a regulatory factors in stem cells. Int $\mathrm{J}$ Stem Cells 12(1):8-20, 2019

9. Lewy-Trenda I, Omulecka A, Janczukowicz J, Papierz W: CD44 Expression in human meningiomas: An immunohistochemical analysis. Pol J Pathol 55(1):33-37, 2004

10. Louis DN, Perry A, Reifenberger G, Deimling AV, FigarellaBranger D, Cavenee WK, et al: The 2016 World Health Organization Classification of Tumors of the central nervous system: A summary. Acta Neuropathol 131(6):803-820, 2016

11. Marta GN, Correa SF, Teixeira MJ: Meningioma: Review of the literature with emphasis on the approach to radiotherapy. Expert Rev Anticancer Ther 11(11):1749-1758, 2011

12. Maynard MA, Ohh M: The role of hypoxia-inducible factors in cancer. Cell Mol Life Sci 64: 2170-2180, 2007

13. McCarty KS, Miller LS, Cox EB, Konrath J, McCarty KS: Estrogen receptor analyses. correlation of biochemical and immunohistochemical methods using monoclonal antireceptor antibodies. Arch Pathol Lab Med 109:716-721, 1985

14. Mojsilovic-Petrovic J, Callaghan D, Cui H, Dean C, Stanimirovic DB, Zhang W: Hypoxia-inducible factor-1 (HIF-1) is involved in the regulation of hypoxia-stimulated expression of monocyte chemoattractant protein-1 (MCP-1/CCL2) and MCP-5 (Ccl12) in astrocytes. J Neuroinflammation 4:12, 2007

15. Mostafa RR, Khairy RA: CD44 Expression in meningioma and its correlation with proliferation indices. J Clin Diagn Res 11(8):12-15, 2017

16. Pakravan N: Tumorigenesis: Cell defense against hypoxia? Oncol Rev 7(1):1-6, 2013 
17. Ponta H, Sherman L, Herrlich PA: CD44: from adhesion molecules to signaling regulators. Nat Rev Mol Cell Biol 4(1):33-35, 2003

18. Reszec J, Hermanowicz A, Rutkowski R, Bernaczyk P, Mariak Z, Chyczewski L: Evaluation of mast cells and hypoxia inducible factor-1 expression in meningiomas of various grades in correlation with peritumoral brain edema. J Neurooncol 115:119-125, 2013

19. Reszec J, Rutkowski R, Chyczewski L: The expression of hypoxia-inducible factor- 1 in primary brain tumors. Int $\mathrm{J}$ Neurosci 123(9):657-662, 2013

20. Sainio M, Zhao F, Heiska L, Turunen O, Bakker MD, Zwarthoff $E$, Lutchman M, Rouleau GA, Jääskeläinen J, Vaheri A, Carpén O: Neurofibromatosis 2 tumor suppressor protein colocalizes with ezrin and CD44 and associates with actin-containing cytoskeleton. J Cell Sci 110:2249-2260, 1997

21. Semenza GL: Hypoxia-inducible factors in physiology and medicine. Cell 148:399-408, 2012
22. Snyder V, Reed-Newman TC, Arnold L, Thomas SM, Anant S: Cancer stem cell metabolism and potential therapeutic targets. Front Oncol 8:203, 2018

23. Xiao ZY, Chen XJ, Pan Q, Yang QZ, Li KZ: Expression of Nestin, CD133 and Sox2 in meningiomas. Turk Neurosurg e1e5, 2018

24. Vaupel P: Hypoxia and aggressive tumor phenotype: Implications for therapy and prognosis. Oncologist 13(3):2126, 2008

25. Vaupel P, Harrison L: Tumor hypoxia: Causative factors, compensatory mechanisms, and cellular response. Oncologist 9(5):4-9, 2004

26. Victorio, Indharty RS, Japardi I: Correlation between hypoxia Inducible factor-1 alpha expression with hystopathological level on intracranial meningiomas patients at RSUP Haji Adam Malik Hospital Medan. Int J PharmTech Res 11(1):49-56, 2018

27. Zhong L: CD133: A stem cell biomarker and beyond. Exp Hematol Oncol 2(17):8, 2013 